\title{
Mathematical model of nonstationary hydraulic processes in gas centrifuge cascade for separation of multicomponent isotope mixtures
}

\author{
Alexey Orlov ${ }^{1}$, Anton Ushakov ${ }^{1,2,}$, and Victor Sovach $^{2}$ \\ ${ }^{1}$ National Research Tomsk Polytechnic University, 634050 Tomsk, Russia \\ ${ }^{2}$ SC "PA Electrochemical Plant", 663690 Zelenogorsk, Russia
}

\begin{abstract}
The article presents results of development of a mathematical model of nonstationary hydraulic processes in gas centrifuge cascade for separation of multicomponent isotope mixtures. This model was used for the calculation parameters of gas centrifuge cascade for separation of silicon isotopes. Comparison of obtained values with results of other authors revealed that developed mathematical model is adequate to describe nonstationary hydraulic processes in gas centrifuge cascades for separation of multicomponent isotope mixtures.
\end{abstract}

\section{Introduction}

Nonstationary hydraulic processes can appear in the operation of gas centrifuge (GC) cascade for separation of multicomponent isotope mixtures (MCIM). During these processes values of pressure and flows of process gas changes in stages of cascade and it is necessary to minimize losses of cascade productivity. At present, there is mathematical model of nonstationary hydraulic processes [1], which has such disadvantage as a limited field of application: modeling is only possible in the case of long cascade and short time periods, when disturbances have not come at withdrawal stages of cascade. Using of this model is impossible for the modeling of nonstationary hydraulic processes in short cascades. For elimination of this disadvantage we have developed the mathematical model of nonstationary hydraulic processes in GC cascade for MCIM separation. This article is devoted to its description.

\section{The description of the mathematical model}

MCIM separation takes place into a cascade (Fig. 1), consisting of $S$ separation stages numbered by $i$ index $(i=\overline{1, S})$ and connected by counter-current type [2]. The cascade has three flows: input feed flow $F$, output light fraction flow $P$ and heavy fraction flow $W$.

\footnotetext{
* Corresponding author: ushakovaa2015@,sibmail.com
} 


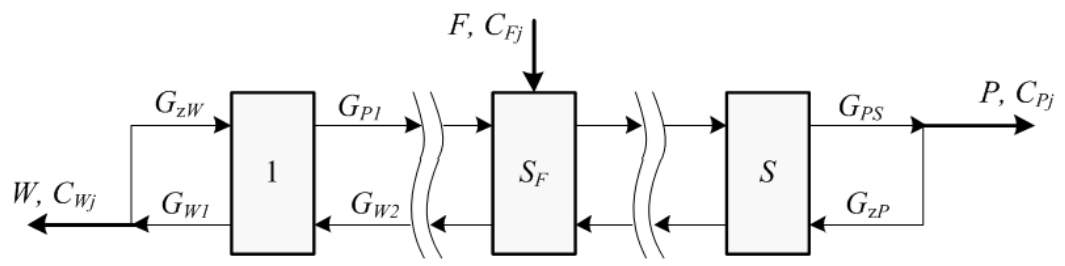

Fig. 1. Separation cascade.

During stationary hydraulic process the flows of cascade are interrelated by material balance equation:

$$
F=P+W
$$

The stage has three flows: input feed flow $G_{F i}$, output light fraction flow $G_{P i}$ and heavy fraction flow $G_{W i}$ :

$$
G_{F i}=G_{P i}+G_{W i}
$$

For square cascade there are dimensionless parameters [3]:

$$
\begin{gathered}
y_{P}=S \ln \chi_{0}, \\
\tau=\frac{G_{F} t \ln ^{2} \chi_{0}}{H^{\prime}},
\end{gathered}
$$

where $y_{P}$ is cascade length; $\chi_{0 i}$ is separation factor per unit difference between the mass numbers; $\tau$ is time; $H^{\prime}$ is cascade holdup, $\mathrm{kg} ; t$ is time, $\mathrm{s}$.

The method of calculation of the nonstationary hydraulic processes is based on the following points:

1. Each stage is composed of finite number of capacities. MCIM is distributed in these capacities.

2. At any time the value of process gas temperature and pressure are the same at any points of capacity.

3. Basic equations of the mathematical model are the balance equations of process gas in each capacity.

In the regards, the holdup of cascade is distributed in $4 S$ capacities (Fig. 2).

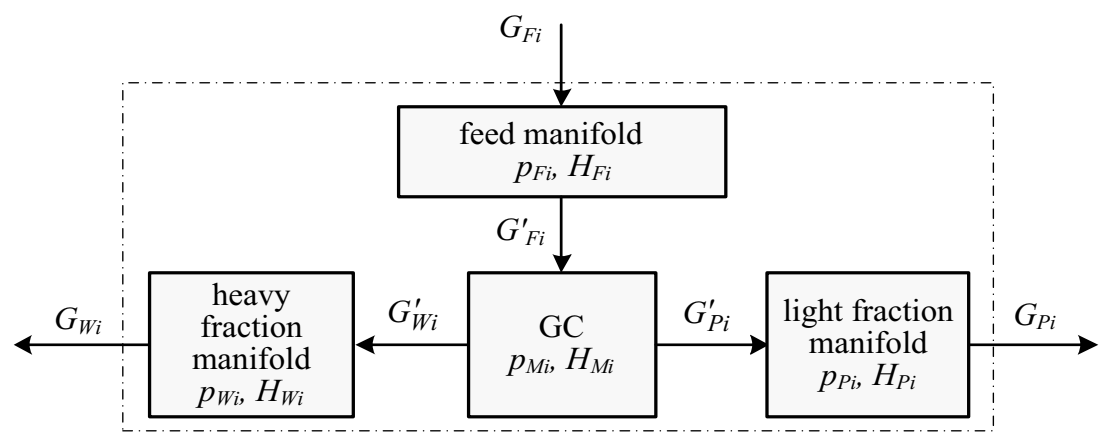

Fig. 2. Capacities of $i$ th stage.

Differential equation describing nonstationary hydraulic processes in capacity which has holdup $H$ and pressure of process gas $p$ is written in the general form as: 


$$
\begin{gathered}
\frac{d H}{d t}=G_{\mathrm{IN}}-G_{\mathrm{OUT}}, \\
H=a \cdot p, \\
a=\frac{\mu V}{R T},
\end{gathered}
$$

where $H$ is capacity holdup, $\mathrm{kg} ; a$ is specific capacity holdup, $\mathrm{kg} \cdot \mathrm{Pa}^{-1} ; \mu$ is molar mass of process gas, $\mathrm{kg} \cdot \mathrm{kmol}^{-1} ; p$ is pressure of process gas, $\mathrm{Pa} ; R=8314 \mathrm{~J} \cdot \mathrm{kmol}^{-1} \cdot \mathrm{K}^{-1}$ is universal gas constant; $G_{\text {IN }}$ is input flow, $\mathrm{kg} \cdot \mathrm{s}^{-1}\left(G_{\mathrm{IN}}=f(p)\right) ; G_{\text {OUT }}$ is output flow, $\mathrm{kg} \cdot \mathrm{s}^{-1}\left(G_{\text {OUT }}=f(p)\right)$.

Replacing derivatives to difference equations by backward Euler method [4], we have received:

$$
\begin{gathered}
a \frac{p^{(k)}-p^{(k-1)}}{\Delta t}=G_{\mathrm{IN}}\left(p^{(k)}\right)-G_{\mathrm{OUT}}\left(p^{(k)}\right), \\
\Delta t=t^{(k)}-t^{(k-1)},
\end{gathered}
$$

where $\Delta t$ is time step; $k$ is the index of time layer.

The calculation have been realized as iterative process: on each iteration cycle calculation of pressure and flows have been done from first stage to $S$-th stage. The initial approximation on $k$-th time layer is the values of pressure and flows on previous $(k-1)$-th time layer. The results of calculation are values of pressure in each capacity and flows at every time layers. We have realized the algorithm described herein as software product, developed on Delphi programming language using Embarcadero Delphi XE2.

\section{The results of calculation cascade for silicon isotope separation}

We have calculated parameters of nonstationary hydraulic process in GC cascade for silicon isotope separation for the purpose of verification of developed mathematical model and created software product. We have seen nonstationary hydraulic process caused by short increase of feed flow of cascade during period of time $\tau=0 \ldots 0,02$. Then initial values of cascade flows have set. The values of relative changes of stage holdup on different time moments which have received by developed model and approximate analytical expression [1] are given on Fig. 3.

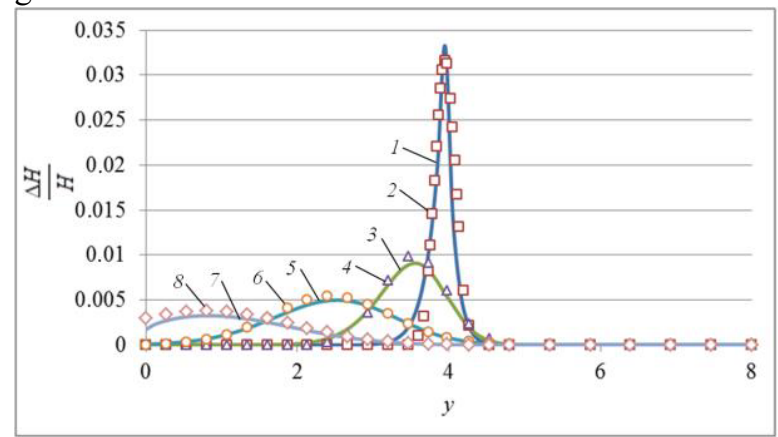

Fig. 3. The values of relative changes of stage holdup on different time moments: 1, 3, 5, 7 developed model; $2,4,6,8-$ the model $[1] ; 1,2-\tau=0,02 ; 3,4-\tau=0,1 ; 5,6-\tau=0,3 ; 7,8-\tau=0,75$. 
As it's seen on Fig. 3, holdup of $S_{F}$-th stage $(y=4)$ has increased. The excess of holdup moves along cascade to stage of withdrawal of heavy fraction flow. Wherein there are insignificant changing of stage holdup located between $S_{F}$-th and $S$-th stages $(y=4 \ldots 5)$ during period of time $\tau=0 \ldots 0,3$.

Thus the results obtained by developed model are well correlated with the results of other researchers [1], which demonstrate the adequacy of developed mathematical model.

\section{Conclusion}

1. The result of research is creation of mathematical model of nonstationary hydraulic processes in gas centrifuge cascade for separation of multicomponent isotope mixtures, representing the differential equation system of first order with given initial conditions.

2. The solution algorithm of equation system describing nonstationary hydraulic processes is developed and realized as a software product.

3. We have made the calculation parameters of nonstationary hydraulic process in gas centrifuge cascade for silicon isotope separation for the purpose of verification of developed mathematical model and created software product. Comparison of obtained values with results of other authors revealed that developed mathematical model is adequate to describe nonstationary hydraulic processes in gas centrifuge cascade for separation of silicon isotope separation.

\section{References}

1. D.M. Levin, Yu.P, Zabelin, Physical and chemical processes during separation of atoms, molecules and in laser, plasma and nanotechnologies conference report collection (CRIatominform, Moscow, 2008)

2. K. Cohen, The theory of isotope separation as applied to the large scale production of $U$ (McGraw Hill, New York, 1951)

3. G.A. Sulaberidze, V.A. Palkin, V.D. Boricevich, V.D. Borman, A.V. Tichomirov, Theory of cascades for separation of binary and multicomponent isotope mixtures (MEPhI, Moscow, 2011)

4. U.G. Pirumov, Numerical methods (Drofa, Moscow, 2003) 